Matherial and methods

This 3-year cross sectional study was performed in Dr. Sheikh Children's Hospital in Mashhad on 50 children with ALL ( $\mathrm{n}=$ $25)$ and NHL $(n=25)$. Half of them were received $(n=25)$ chemotherapy alone and half of them chemotherapy plus radiotherapy $(n=25)$. All children were in the remission phase. We assessed them by DEXA bone mineral densitometery (BMD) on the lumbar spine and femoral neck (hip). We also measured some bone biomarkers include calcium (ca), phosphorus (p), parathoromone (PTH), alkaline phosphatase (ALP) in plasma. Results by age, height, sex and Body Mass Index (BMI) were adjusted with a special software.

Results Mean age was $8.28 \pm 3.93$ years. There was no significant difference on bone biomarkers (Ca, P, ALP. PTH) between ALL, NHL and also between the two treatment groups. Children with ALL had lower density at the hip and lumbar spine. (respectively $\mathrm{p}$ value $<0.001$ and $\mathrm{p}$ value $=0.018$ ). A total of 50 patients, the hip BMD showed normal results in 3 patients $(6 \%)$, in 14 patients (28\%) osteopenia were seen and 33 patients $(66 \%)$ had osteoporosis. In whom received radiotherapy plus chemotherapy, one patient had normal BMD and 24 patients (48\% of total patients) at the hip and 22 patients (44\%) at lumbar spine had decreased BMD. In contrast, in whom had only chemotherapy, 24 patients (48\%) had osteoporosis at hip and 23 $(46 \%)$ at the lumbar spine. There was no significant difference in BMD between the sexes.

Conculsion Given that 94\% of children had abnormal bone density, Seem to pay more attention to the metabolic status and BMD in children with cancer can develop appropriate strategies to improve health and quality of their life.

\section{PO-0158 MANAGEMENT OF PATIENTS WITH ALL WHEN EXPOSED TO VZV}

C Glancy, R Kennedy. Paediatrics, Craigavon Area Hospital, Belfast, UK

\subsection{6/archdischild-2014-307384.820}

Aims Investigate the management of patients with ALL when exposed to Varicella Zoster Virus.

Method Retrospective study looking at all patients diagnosed with ALL between 2007-2011, a total of 60 patients. Each chart was looked at for any documented exposure to varicella and the management of the patient compared to standards set on local guidelines.

Results 58 patients in the audit. Age range was from 2 years to 15 years. 48 patients were tested at diagnosis leaving 10 patients with unknown Varicella status diagnosis.

24 patients reported exposure, 50\% of these patient exposures were significant and required treatment. 19 of these were managed appropriately and 5 were not. $100 \%$ patients that were exposed and found not to be significant exposures did not receive treatment and therefore were managed appropriately. Of the patients that were exposed and not managed according to local guidance, one patient received IVIG 2/52 after a significant exposure when according to guidance they should have received oral aciclovir. Another patient did not have their status checked at diagnosis or when exposed. The remaining three patients did not have their immune status checked at diagnosis making their management inappropriate.

Conclusion Overall the management of the patients who contacted the medical team to report exposure to Varicella were managed appropriately. Plan is to have a sticker on the front of patient notes with varicella status on diagnosis, exposure and results.

\section{P0-0159 CHILDHOOD DISSEMINATED RHABDOMYOSARCOMA OF UNKNOWN PRIMARY SITE MASQUERADING AS AN ACUTE HAEMATOLOGICAL MALIGNANCY- LITERATURE REVIEW APROPOS OF TWO UNUSUAL CASES}

${ }^{1} \mathrm{G}$ Karpinsky, ${ }^{1} \mathrm{E}$ Rak, ${ }^{2} \mathrm{M}$ Krawczyk, ${ }^{2} \mathrm{E}$ Bien. ${ }^{1}$ English Division Pediatric Oncology Scientific Circle, Medical University of Gdansk, Gdansk, Poland; '²Department of Pediatrics Hematology and Oncology, Medical University of Gdansk, Gdansk, Poland

\subsection{6/archdischild-2014-307384.821}

Background Small round cell tumours, particularly rhabdomyosarcoma (RMS), may infiltrate bone marrow (BM), mimicking acute leukaemia - both clinically and in morphological assessment of myelogram.

Aim To analyse diagnostic and therapeutic dilemmas in children with RMS masquerading as acute leukaemia.

Methods A retrospective analysis of medical charts of a 14-yearold male and 15-year-old female admitted to Department of Paediatrics, Haematology and Oncology, Medical University of Gdansk, Poland in 2007 and 2013 and literature review.

Results Both patients were referred suspected of acute leukaemia presenting with weakness, pallor, bone pains, and enlarged peripheral lymph nodes (LN). Skin bruising, petechiae and wound bleedings were progressing. Laboratory tests showed anaemia, thrombocytopenia and features of acute DIC and ATLS. BM aspiration revealed blast cells suggestive for leukaemia. Flow cytometry failed to display lymphoid or myeloid antigens. Aspirational LN biopsy revealed small round blue cells, suggesting AML, non-Hodgkin's lymphoma or Ewing's sarcoma. Modified chemotherapy in all these malignancies was introduced to alleviate DIC-associated haemorrhages and enable LN resection. Finally the diagnoses of embryonal RMS (male) and alveolar RMS (female) were made. Administration of proper chemotherapy for metastatic RMS resulted in rapid neoplasms' regression and normalisation of DIC parameters.

Conclusion Clinical presentation of childhood RMS masquerading as acute leukaemia is unique and poses diagnostic problems, especially in patients with DIC-related haemorrhages. RMS should be included in differential diagnosis of any case presenting as a systemic disease with BM infiltration of cells mimicking leukemic blasts, but lacking lymphoid and myeloid antigens in immunophenotyping by flow cytometry.

\section{P0-0160 INFECTIOUS EVENTS DURING INTENSIVE TREATMENT IN CHILDHOOD ACUTE LYMPHOBLASTIC LEUKAEMIA}

${ }^{1} \mathrm{~K}$ Martinova, ${ }^{1} \mathrm{~B}$ Coneska-Jovanova, ${ }^{1} \mathrm{~S}$ Kocheva, ${ }^{1} \mathrm{~A}$ Jovanovska, ${ }^{2} \mathrm{E}$ Bojadgieva. ${ }^{1}$ Hematology and Oncology, University Children's Hospital, Skopje, Macedonia; ${ }^{2}$ Faculty of Medical Sciences, University Goce Delcev, Stip, Macedonia

\subsection{6/archdischild-2014-307384.822}

Background Many children with acute lymphoblastic leukaemia (ALL) experience one or more infectious complications during treatment. Infections are important to study in children with ALL because they continue to contribute to morbidity and mortality, affect quality of life for children and their families and require considerable health resources to prevent and treat.

Aims To analyse the characteristics of infective episodes (I. E.) during intensive treatment (Protocol I, M and II) in children with ALL.

Methods Objective of this study ware 55 patients with ALL who were treated according to ALL-BFM 90 and ALL-BFM 95 Protocol between January 2000 and December 2007 at the University 
Children's Hospital in Skopje. We explored the characteristics of I.E., together with the causative pathogens, the episodes of febrile neutropenia (FN), the length of antibiotic treatments and the treatments with G-CSF during intensive phases of treatment (Protocol I, M and II).

Results From 55 analysed records 24 (43.64\%) were male and $31(56.36 \%)$ were female. Mean age at diagnosis was 6.0 years (1.1-15.0). Majority of the patients $43(78 \%)$ were under 10 years and $12(22 \%)$ were over 10 years. All of them experienced 132, 52 and 73 I. E. with 2.4, 0.9, and 1.3 infections per patient during Protocol I, M and II respectively. Regarding to the pathogens 184 (71.5\%) were bacterial (102, 30 and 52 in Protocol I, $\mathrm{M}$ and II), 45 (17.5\%) were viral (20, 14 and 11 in Protocol I, $\mathrm{M}$ and II) and $28(10.8 \%)$ were fungal $(10,8,10$ in the three intensive phases respectively). There was a slight predominance of gram positive bacteria in Protocol I [Gram positive 42 $(51.85 \%)$ versus gram negative 34 (41.97\%)], and a very slight predominance of gram negative bacteria in Protocol II [Gram positive 16 (45.71\% versus Gram negative 18 (51.42\%)]. The infections were treated with antibiotic treatment in average of 23.69, 11 and 15.05 days and the number of treatments with G-CSF were in average 7.22, 2.44 and 9.20 per patient respectively in Protocol I, M and II. The number of episodes of FN in these three phases was 16.4 (29.1\%), 4 (7.3\%) and 22 (40\%).

Summary/conclusion Evaluation of the characteristics of I. E. presented that the majority of infectious events were observed in Protocol I and also the length of antibiotic treatment was longer in this phase. But the episodes of FN together with the treatments with G-CSF were higher in Protocol II possible due to the cumulative effect of chemotherapy.

\section{PO-0161 TREATMENT OF IRON OVERLOAD WITH DEFERASIROX IN THE PATIENTS WITH THALASSAEMIA MAJOR}

${ }^{1} \mathrm{~K}$ Martinova, ${ }^{1} \mathrm{~B}$ Coneska-Jovanova, 'S Kocheva, ${ }^{1} \mathrm{~A}$ Jovanovska, ${ }^{2} \mathrm{E}$ Bojadgieva. ${ }^{1}$ Hematology and Oncology, University Children's Hospital, Skopje, Macedonia; ${ }^{2}$ University Goce Delcev, Faculty of Medical Sciences, Stip, Macedonia

\subsection{6/archdischild-2014-307384.823}

Background Iron chelation is an important component of management of transfusion-dependent patients with thalassaemia major. Deferasirox is a relatively new oral iron chelator (US FDA approved in 2005) with the limited experience in children.

Aims To present our experience with deferasirox in patients with thalassaemia major (TM) in the context of: effects on serum ferritin level in chronically transfused patients with thalassaemia major, side effects and patients tolerance to the drug and effects on serum creatinin and liver transaminases.

Methods Four patients with TM with mean age of 3,1 years (range 2,5-3,5) were included in the study. Mean follow up was 37 months (range 29-42). The disease was diagnosed in early childhood (during the first year of life) with the following signs and symptoms: extreme pallor, jaundice, failure to thrive, poor feeding, irritability, decreased activity and hepatosplenomegaly. Regular blood transfusions were applied to treat chronic hemolytic anaemia.

Results The mean serum ferritin (SF) at diagnosis was 471,3 \pm 284,4 (range 155-706), and at the start of the treatment with deferasirox $6281 \pm 9183,9$ (range 767-20000). The number of blood transfusions before the treatment was around 28,3 $\pm 15,5$ units (range 17-46), or about 679,3 \pm 608,14 (range 220-1369) $\mathrm{ml} / \mathrm{kg}$ body weight. Deferasirox was given seven days a week at a dose of $20 \mathrm{mg} / \mathrm{kg}$ body weight. The primary outcome variable was SF level at the start and at the end of the study. Echocardiography was made in all patients and it was normal. MRI-T2* could not be performed because there was no specific software. The level of SF at the end of the study period was $1862 \pm$ 1312,15 (range 637-3710). Patients were monitored for hepatic and renal toxicity, visual or auditory changes and development of new symptoms. Adverse events were very mild gastrointestinal symptoms in 1 patient and no adverse events in the remaining 3 patients. Elevation of serum creatinin or hepatic transaminases was not observed in any subject. One patient interrupted the therapy as there was a marked fall in SF $<500 \mathrm{ng} / \mathrm{L}$ at the end of the therapy. The treatment was well tolerated; suspension of therapy was not required owing to toxicity.

Summary/conclusion The results suggest that deferasirox is effective in lowering iron burden, it is well tolerated and has a low potential for toxicity. Long term therapy will be needed to asses the benefits on iron balance and organ damage in chronically transfused patients with thalassaemia major.

\section{PO-0162 CLINICAL PROFILE AND OUTCOME OF CHILDREN WITH ANAPLASTIC LARGE CELL LYMPHOMA}

'L Mathew, ${ }^{1} D$ Boddu, ${ }^{1} \mathrm{R}$ John, ${ }^{1} \mathrm{~N}$ Chaudhry, ${ }^{2} \mathrm{M}$ Theres. ${ }^{1}$ Child Health, Christian Medical College Hospital, Vellore, India; ${ }^{2}$ Pathology, Christian Medical College Hospital, Vellore, India

\subsection{6/archdischild-2014-307384.824}

Background Anaplastic large cell lymphoma (ALCL) in children is usually ALK positive and is characterised by advanced disease at presentation with a high incidence of extra nodal involvement. We present the clinical profile, treatment and outcome of a small cohort of children with ALCL treated at our centre between January 2004 and June 2013.

Results 19 children, 14 boys and 5 girls, aged 18 months to 14 years, with ALCL. 16/19 had lymphadenopathy and 14/19 had fever. Thirteen had high risk, 5 had standard risk and one had low risk disease. 16/19 ALK positive. 17/19 were treated according to the NHL-BFM 90 protocol. Fourteen completed treatment. Three died during treatment; 2 due to infection and 1 had progressive disease. All 3 had high risk disease and were ALK positive. All thethree children with ALK negative disease relapsed; one died on relapse treatment, another was lost to follow up a year after completion of relapse therapy and the third with primary cutaneous disease, is alive and well. Of the 11 ALK positive children who completed treatment, only one relapsed and died. The remaining 10 are alive and well with a mean follow up of 17 months (range 8-93 months).

Conclusion The majority of our patients are ALK positive and have high risk disease at presentation. 55\% of children with high risk disease are alive and well after a mean follow up of 17 months. The overall survival was $65 \%$ and event free survival $58 \%$ at the time of this analysis.

\section{PO-0163 EFFECT OF NUTRITION WITH FOLLOW-UP FORMULA FROM 6TH MONTH OF AGE ON THE COMPLETE BLOOD COUNT AND IRON LEVELS OF INFANTS (PRELIMINARY TRIAL)}

H Apak, N Ozdemir, G Tuysuz, B Kutlubay, E Erginoz, M Kucur. Pediatric Hematology, Cerrahpasa Medical Faculty University of Istanbul, Istanbul, Turkey

10.1136/archdischild-2014-307384.825 